\title{
A note on the Peterson hit problem for the Steenrod algebra
}

\author{
By Nguyen Khac Tin \\ Faculty of Applied Sciences, Ho Chi Minh City University of Technology and Education, 01 Vo Van Ngan, Linh \\ Chieu, Thu Duc, Ho Chi Minh, Vietnam \\ (Communicated by Kenji Fukaya, M.J.A., March 12, 2021)
}

\begin{abstract}
Let $P_{k}=\oplus_{n \geqslant 0}\left(P_{k}\right)_{n} \cong \mathbf{F}_{2}\left[x_{1}, x_{2}, \ldots, x_{k}\right]$ be the graded polynomial algebra over the prime field of two elements $\mathbf{F}_{2}$, in $k$ generators $x_{1}, x_{2}, \ldots, x_{k}$, each of degree 1 . Being the mod-2 cohomology of the classifying space $B(\mathbf{Z} / 2)^{k}$, the algebra $P_{k}$ is a module over the mod-2 Steenrod algebra $\mathcal{A}$.

In this Note, we explicitly compute the hit problem of some generic degrees $r\left(2^{s}-1\right)+2^{s} m$ in $P_{k}$, where $r=k-1=4, m \in\{8 ; 10 ; 11\}$ and $s$ an arbitrary non-negative integer. Moreover, as a consequence, we get the dimension results for polynomial algebra in some generic degrees and in the cases $k=5$ and 6 .
\end{abstract}

Key words: Polynomial algebra; Steenrod algebra; hit problem.

1. Introduction. Denote by $P_{k}=$ $H^{*}\left(\left(\mathbf{R} P^{\infty}\right)^{k}\right)$ the modulo-2 cohomology algebra of the direct product of $k$ copies of infinite dimensional real projective spaces $\mathbf{R} P^{\infty}$. Then, $P_{k}$ is isomorphic to the graded polynomial algebra $\mathbf{F}_{2}\left[x_{1}, x_{2}, \ldots, x_{k}\right]$ of $k$ variables, in which each $x_{j}$ is of degree 1 . Here the cohomology is taken with coefficients in the prime field $\mathbf{F}_{2}$ of two elements.

The $\mathcal{A}$-module structure of $P_{k}$ is explicitly determined by the formula

$$
S q^{i}\left(x_{j}\right)= \begin{cases}x_{j}, & i=0 \\ x_{j}^{2}, & i=1 \\ 0, & i>1\end{cases}
$$

and the Cartan formula $S q^{n}(x y)=$ $\sum_{i=0}^{n} S q^{i}(x) S q^{n-i}(y)$, where $x, y \in P_{k}$ (see Steenrod and Epstein [18]).

A polynomial $f$ in $P_{k}$ is called hit if it can be written as a finite sum $f=\sum_{u \geqslant 0} S q^{2^{u}}\left(h_{u}\right)$ for suitable polynomials $h_{u}$. That means $f$ belongs to $\mathcal{A}^{+} P_{k}$, where $\mathcal{A}^{+}$denotes the augmentation ideal in $\mathcal{A}$.

The Peterson hit problem is to find a minimal generating set for $P_{k}$ regarded as a module over the mod-2 Steenrod algebra. Equivalently, this problem is to find a basis for the vector space

$$
\mathbf{F}_{2} \otimes_{\mathcal{A}} P_{k} \cong P_{k} / \mathcal{A}^{+} P_{k}
$$

2010 Mathematics Subject Classification. Primary 55S10, 55S05, 55T15. in each degree $n$, where $\mathcal{A}^{+}$is an ideal of $\mathcal{A}$ generated by all Steenrod squares of positive degrees. Such a basis may be represented by a list of monomials of degree $n$.

This problem has first been studied by Peterson [10], Wood [28], Singer [16], Priddy [14], who pointed out its relationship with some classical problems in homotopy theory such as the cobordism theory of manifolds, the modular representation theory of linear groups, Adams spectral sequences of stable homotopy of spheres, and stable homotopy type of the classifying space of finite groups. Then, this problem was investigated by Wood [28], Carlisle and Wood [1], Silverman [17], Nam [8,9], Mothebe [7], Sum [19,21], Cho'n and Hà [3], Kameko $[5,6]$ and others. Recently, the hit problem and its applications to representations of general linear groups have been presented in the books of Walker and Wood [26,27].

For a positive integer $n$, by $\mu(n)$ one means the smallest number $r$ for which it is possible to write $n=\sum_{1 \leqslant i \leqslant r}\left(2^{u_{i}}-1\right)$, where $u_{i}>0$. This result implies a result of Wood, which originally is a conjecture of Peterson [10].

Theorem 1.1 (See Wood [28]). If $\mu(n)>k$, then $\left(\mathbf{F}_{2} \otimes_{\mathcal{A}} P_{k}\right)_{n}=0$.

Let $G L_{k}$ be the general linear group over the field $\mathbf{F}_{2}$. This group acts naturally on $P_{k}$ by matrix substitution. Since the two actions of $\mathcal{A}$ and $G L_{k}$ upon $P_{k}$ commute with each other, there is an action of $G L_{k}$ on $\mathbf{F}_{2} \otimes_{\mathcal{A}} P_{k}$. One of our main tools is 
Kameko's homomorphism $\widetilde{S q_{*}^{0}}: \mathbf{F}_{2} \otimes_{\mathcal{A}} P_{k} \rightarrow$ $\mathbf{F}_{2} \otimes_{\mathcal{A}} P_{k}$, which is induced by an $\mathbf{F}_{2}$-linear map $\phi_{k}: P_{k} \rightarrow P_{k}$, given by

$$
\phi_{k}(x)= \begin{cases}y, & \text { if } x=x_{1} x_{2} \ldots x_{k} y^{2} \\ 0, & \text { otherwise }\end{cases}
$$

for any monomial $x \in P_{k}$. The map $\phi_{k}$ is not an $\mathcal{A}$-homomorphism. However, $\phi_{k} S q^{2 i}=S q^{i} \phi_{k}$ and $\phi_{k} S q^{2 i+1}=0$ for any non-negative integer $i$.

Theorem 1.2 (Kameko [4]). Let d be a nonnegative integer. If $\mu(2 d+k)=k$, then

$$
\widetilde{S q_{*}^{0}}:\left(\mathbf{F}_{2} \otimes_{\mathcal{A}} P_{k}\right)_{2 d+k} \longrightarrow\left(\mathbf{F}_{2} \otimes_{\mathcal{A}} P_{k}\right)_{d}
$$

is an isomorphism of $G L_{k}$-modules.

From the results of Wood [28] and Kameko [4], the hit problem is reduced to the case of degree $n$ of the form

$$
n=r\left(2^{s}-1\right)+2^{s} m
$$

where $r, s, m$ are non-negative intergers such that $1 \leqslant r<k$ and $\mu(m)<r$.

For $r=k-1$ and $m>0$, the problem was studied by Crabb and Hubbuck [2], Nam [8], Repka and Selick [15], Walker and Wood [25], Sum [21].

Now, the $\mathbf{F}_{2}$-vector space $\mathbf{F}_{2} \otimes_{\mathcal{A}} P_{k}$ was explicitly calculated by Peterson [10] for $k=1,2$, by Kameko [4] for $k=3$ and by Sum [20,21] for $k=4$. However, for $k>4$, it is still unsolved, even in the case of $k=5$ with the help of computers.

For $r=k-1=4$ and $m=0$, the vector space $\left(\mathbf{F}_{2} \otimes_{\mathcal{A}} P_{5}\right)_{n}$ is explicitly computed by Phuc and Sum $[11,12]$, and in the case $r=k-2=3, m=1$ by Phuc [13]. In the present paper, we study the hit problem of some generic degrees $r\left(2^{s}-1\right)+2^{s} m$ in $P_{k}$, where $r=k-1=4, m \in\{8 ; 10 ; 11\}$ and $s$ an arbitrary non-negative integer. Moreover, as a consequence, we get the dimension results for polynomial algebra in some generic degrees and in the cases $k=5$ and 6 .

The proofs of the results of this Note will be published in detail elsewhere.

2. The Main Results. From now on, we denote by $B_{k}(n)$ the set of all admissible monomials of degree $n$ in $P_{k}$. The following is one of our main results.

Theorem 2.1. Let $n=(k-1) \cdot\left(2^{s}-1\right)+$ $11.2^{s}$ with $s$ an arbitrary non-negative integer. For $k=5$, then

$$
\operatorname{dim}\left(\mathbf{F}_{2} \otimes_{\mathcal{A}} P_{5}\right)_{4\left(2^{s}-1\right)+11.2^{s}}= \begin{cases}315, & \text { if } s=0, \\ 1024, & \text { if } s=1, \\ 1984, & \text { if } s \geqslant 2 .\end{cases}
$$

Note that this theorem has been proved by Mothebe [7] for $s=0$, and by Walker and Wood [26] for $s=1$, and by another method.

The proof of the above theorem is too long and very technical. It is proved by explicitly determining all admissible monomials of degree $n=4\left(2^{s}-\right.$ 1) $+11.2^{s}$ in $P_{5}$, for any $s \geqslant 0$.

Sketch proof of Theorem 2.1. We first recall some notations and definitions in [21]. For any $1 \leqslant i \leqslant k$, define the homomorphism $f_{i}$ : $P_{k-1} \rightarrow P_{k}$ of algebras by substituting

$$
f_{i}\left(x_{j}\right)= \begin{cases}x_{j}, & \text { if } 1 \leqslant j<i, \\ x_{j+1}, & \text { if } i \leqslant j<k .\end{cases}
$$

Then, $f_{i}$ is a homomorphism of $\mathcal{A}$-modules.

Denote $I=\left\{i_{1}, i_{2}, \ldots, i_{r}\right\}$ and $\mathcal{U}_{k}=\left\{(i ; I): 1 \leqslant i<i_{1}<\ldots<i_{r} \leqslant k, 0 \leqslant r<k\right\}$.

Let $\quad(i ; I) \in \mathcal{U}_{k}, \quad x_{(I, u)}=x_{i_{u}}^{2^{r-1}+\ldots+2^{r-u}} \Pi_{u<t \leqslant r} x_{i_{t}}^{2^{r-t}}$ for $r=\ell(I)$ be the length of $I, x_{(\varnothing, 1)}=1$. For a monomial $x \in P_{k-1}$, we define the monomial $\varphi_{(i ; I)}(x)$ in $P_{k}$ by setting

$\varphi_{(i ; I)}(x)= \begin{cases}\left(x_{i}^{2^{r}-1} f_{i}(x)\right) / x_{(I, u)} & \text { if there exists } u \\ & \text { such that } x \text { is } \\ & u \text {-compatible with } \\ & (i, I), \\ 0, & \text { otherwise. }\end{cases}$

Then we have an $\mathbf{F}_{2}$-linear map $\varphi_{(i ; I)}: P_{k-1} \rightarrow$ $P_{k}$.

For $k=5$, we have $n=(k-1)\left(2^{s}-1\right)+2^{s} m=$ $\sum_{i=0}^{3}\left(2^{s+i}-1\right)$ and set $d_{1}=s+3, d_{2}=-s+2, d_{3}=$ $s+1, d_{4}=s$. Thus, using the results in Sum [21] we see that $B_{5}\left(4 .\left(2^{s}-1\right)+11.2^{s}\right)=\Phi\left(B_{4}\left(4 .\left(2^{s}-1\right)+\right.\right.$ $\left.11.2^{s}\right)$ ) is a minimal set of generators for $\mathcal{A}$-module $P_{5}$ in degree 4. $\left(2^{s}-1\right)+11.2^{s}$ for any $s \geqslant 4$. Here,

$$
\Phi\left(B_{4}(n)\right)=\Phi^{0}\left(B_{4}(n)\right) \cup \Phi^{+}\left(B_{4}(n)\right)
$$

where

$\Phi^{0}\left(B_{4}(n)\right)=\cup_{1 \leqslant i \leqslant 5} \varphi_{(i ; \varnothing)}\left(B_{4}(n)\right)=\cup_{1 \leqslant i \leqslant 5} f_{i}\left(B_{4}(n)\right)$,

$\Phi^{+}\left(B_{4}(n)\right)=\cup_{(i ; I) \in \mathcal{U}_{5}, 0<\ell(I) \leqslant 4} \varphi_{(i ; I)}\left(B_{4}(n)\right) \backslash P_{5}^{0}$,

$P_{5}^{0}=\left\langle\left\{x=x_{1}^{a_{1}} \ldots x_{5}^{a_{5}}: a_{1} a_{2} \ldots a_{5}=0\right\}\right\rangle$.

Moreover, we set $m=2^{d_{1}-d_{4}}+2^{d_{2}-d_{4}}+2^{d_{3}-d_{4}}-$ $3=11$, using the result in Sum [21] one get 


$$
\left|B_{5}(n)\right|=\left(2^{5}-1\right) \cdot\left|B_{4}(11)\right| \text { for any } s \geqslant 4 .
$$

Based on the result in [21], we obtain $\left(\mathbf{F}_{2} \otimes_{\mathcal{A}} P_{4}\right)_{11}=$ 64. And therefore, $\operatorname{dim}\left(\mathbf{F}_{2} \otimes_{\mathcal{A}} P_{5}\right)_{4 .\left(2^{s}-1\right)+11.2^{s}}=1984$, for any $s \geqslant 4$. Hence, we need only to prove the theorem for $s=0,1,2,3$ by the direct computations. The proof of the theorem in these cases is too long and very technical. It is proved by explicitly determining all admissible monomials of degree $n=$ $4\left(2^{s}-1\right)+11.2^{s}$ in $P_{5}$, for $s \leqslant 3$. The ideas of proofs are from Kameko's squaring operation [4] combining with the results in Sum $[19,21]$. Note that the results dimension of $\left(\mathbf{F}_{2} \otimes_{\mathcal{A}} P_{5}\right)_{4 .\left(2^{s}-1\right)+11.2^{s}}, 2 \leqslant s \leqslant 3$ has been verified by using a computer calculation program of V. H. Vu in SAGE. I would like to say thank you for his support.

Moreover, from the results of this theorem and in Sum [21], we get the dimension results for polynomial algebra of six variables as follows:

Corollary 2.2 (Tin [23]). For any integer $d>5$, there exist exactly 19845 admissible monomials of degree $m_{1}=5\left(2^{d}-1\right)+3.2^{d}$ in $P_{6}$. Consequently,

$$
\operatorname{dim}\left(\mathbf{F}_{2} \otimes_{\mathcal{A}} P_{6}\right)_{m_{1}}=19845 .
$$

Corollary 2.3 (Tin [24]). For any $\ell>4$, there exist exactly 64512 admissible monomials of degree $m_{2}=5\left(2^{\ell}-1\right)+13.2^{\ell+1}$ in $P_{6}$. Consequent$l y, \operatorname{dim}\left(\mathbf{F}_{2} \otimes_{\mathcal{A}} P_{6}\right)_{m_{2}}=64512$.

It is easy to check that $\mu(56)=4=\alpha(56+$ $\mu(56))=\alpha(60)$ and we have $m_{2}=5\left(2^{t}-1\right)+$ $7.2^{t+3}=5\left(2^{t}-1\right)+56.2^{t}$ hence, using the results in $[21]$ one get

$\left|B_{6}\left(5\left(2^{t}-1\right)+7.2^{t+3}\right)\right|=\left(2^{6}-1\right)\left|B_{5}(56)\right|=124992$, for any integer $t \geqslant k-1=5$. We get the corollary following

Corollary 2.4. There exist exactly 124992 admissible monomials of degree $m_{3}=5\left(2^{t}-1\right)+$ $7.2^{t+3}$ in $P_{6}$, for any $t \geqslant 5$. Consequently, $\operatorname{dim}\left(\mathbf{F}_{2} \otimes_{\mathcal{A}} P_{6}\right)_{m_{3}}=124992$.

By a simple calculation, we have $\mu(116)=4$ and $\alpha(116+\mu(116))=4$. Using the results in [21] one get the corollary following

Corollary 2.5. For any integer $r>4$, there exist exactly 124992 admissible monomials of degree $m_{4}=5\left(2^{r}-1\right)+29.2^{r+2} \quad$ in $\quad P_{6} . \quad$ Consequently, $\operatorname{dim}\left(\mathbf{F}_{2} \otimes_{\mathcal{A}} P_{6}\right)_{m_{4}}=124992$.

Set $\quad t(k, d)=\max \{0, k-\alpha(d+k)-\zeta(d+k)\}$ where $\zeta(n)$ the greatest integer $u$ such that $n$ is divisible by $2^{u}$, that means $n=2^{\zeta(n)} m$, with $m$ an odd integer. We recall a result in [22] the following

Theorem 2.6 (Tin and Sum [22]). Let $d$ be an arbitrary non-negative integer. Then

$$
\begin{aligned}
& \left(\widetilde{S q_{*}^{0}}\right)^{s-t}:\left(\mathbf{F}_{2} \otimes_{\mathcal{A}} P_{k}\right)_{k\left(2^{s}-1\right)+2^{s} d} \\
& \quad \longrightarrow\left(\mathbf{F}_{2} \otimes_{\mathcal{A}} P_{k}\right)_{k\left(2^{t}-1\right)+2^{t} d}
\end{aligned}
$$

is an isomorphism of $G L_{k}$-modules for every $s \geqslant t$ if and only if $t \geqslant t(k, d)$.

It is easy to check that for $k=5$ and $d=56$ then

$$
t(k, d)=\max \{0, k-\alpha(d+k)-\zeta(d+k)\}=0 .
$$

Then, from the results of Theorems 2.1 and 2.6 we get the corollary following

Corollary 2.7. For any integer $u \geqslant 0$, there exist exactly 1984 admissible monomials of degree $5\left(2^{u}-1\right)+7.2^{u+3}$ in $P_{5}$. Consequently, $\operatorname{dim}\left(\mathbf{F}_{2} \otimes_{\mathcal{A}} P_{5}\right)_{5\left(2^{u}-1\right)+7.2^{u+3}}=1984$.

Similarly, for $k=5, d=116$ then $t(k, d)=$ $\max \{0, k-\alpha(d+k)-\zeta(d+k)\}=0$. We have the corollary following

Corollary 2.8. For any integer $v \geqslant 0$, there exist exactly 1984 admissible monomials of degree $5\left(2^{v}-1\right)+29.2^{v+2}$ in $P_{5}$. Consequently, $\operatorname{dim}\left(\mathbf{F}_{2} \otimes_{\mathcal{A}} P_{5}\right)_{5\left(2^{v}-1\right)+29.2^{v+2}}=1984$.

Next, we determine the hit problem in degree $n$ of the form (1.1) with $r=k-1=4, m=8$ and $s$ an arbitrary non-negative integer.

Theorem 2.9. Let $n=4 .\left(2^{s}-1\right)+8.2^{s}$ with $s$ an arbitrary non-negative integer. Then

$$
\operatorname{dim}\left(\mathbf{F}_{2} \otimes_{\mathcal{A}} P_{5}\right)_{4 .\left(2^{s}-1\right)+8.2^{s}}= \begin{cases}174, & \text { if } s=0, \\ 641, & \text { if } s=1, \\ 1426, & \text { if } s=2, \\ 1706, & \text { if } s=3, \\ 1705, & \text { if } s \geqslant 4 .\end{cases}
$$

Similarly, the following is our third main result.

Theorem 2.10. Let $n=(k-1) \cdot\left(2^{s}-1\right)+$ $10.2^{s}$ with $s$ an arbitrary non-negative integer. For $k=5$, then

$$
\operatorname{dim}\left(\mathbf{F}_{2} \otimes_{\mathcal{A}} P_{5}\right)_{4\left(2^{s}-1\right)+10.2^{s}}= \begin{cases}280, & \text { if } s=0, \\ 961, & \text { if } s=1, \\ 1905, & \text { if } s=2, \\ 2171, & \text { if } s=3, \\ 2170, & \text { if } s \geqslant 4 .\end{cases}
$$

The ideas of the proofs of Theorems 2.9 and 2.10 are the same as in proof of Theorem 2.1. 
Acknowledgments. I would like to express my warmest thanks to my adviser, Prof. Nguyễn Sum for helpful conversations. I also would like to thank Ho Chi Minh City University of Technology and Education for supporting this work. Finally, the author thank the referee for helpful comments.

\section{References}

[ 1 ] D. P. Carlisle and R. M. W. Wood, The boundedness conjecture for the action of the Steenrod algebra on polynomials, in Adams Memorial Symposium on Algebraic Topology, 2 (Manchester, 1990), 203-216, London Math. Soc. Lecture Note Ser., 176, Cambridge Univ. Press, Cambridge, 1992.

[ 2 ] M. C. Crabb and J. R. Hubbuck, Representations of the homology of $B V$ and the Steenrod algebra. II, in Algebraic topology: new trends in localization and periodicity (Sant Feliu de Guixols, 1994), 143-154, Progr. Math., 136, Birkhäuser, Basel, 1996.

[ 3 ] P. H. Cho'n and L. M. Hà, On May spectral sequence and the algebraic transfer, Proc. Japan Acad. Ser. A Math. Sci. 86 (2010), no. 9, 159-164.

[ 4 ] M. Kameko, Products of projective spaces as Steenrod modules, ProQuest LLC, Ann Arbor, MI, 1990.

[ 5 ] M. Kameko, Generators of the cohomology of $B V_{3}$, J. Math. Kyoto Univ. 38 (1998), no. 3, $587-593$.

[ 6 ] M. Kameko, M. Nakagawa and T. Nishimoto, On the mod 2 cohomology of the classifying space of the exceptional Lie group $E_{6}$, Proc. Japan Acad. Ser. A Math. Sci. 95 (2019), no. 9, 91-96.

[ 7 ] M. Mothebe, Dimensions of the polynomial algebra $\mathbf{F}_{2}\left[x_{1}, \ldots, x_{n}\right]$ as a module over the Steenrod algebra, JP J. Algebra Number Theory Appl. 13 (2009), no. 2, 161-170.

[ 8 ] T. N. Nam, $\mathcal{A}$-générateurs génériques pour l'algèbre polynomiale, Adv. Math. 186 (2004), no. 2, 334-362.

[ 9 ] T. N. Nam, Transfert algébrique et action du groupe linéaire sur les puissances divisées modulo 2, Ann. Inst. Fourier (Grenoble) $\mathbf{5 8}$ (2008), no. 5, 1785-1837.

[ 10 ] F. P. Peterson, Generators of $H^{*}\left(\mathbf{R P}^{\infty} \times \mathbf{R} \mathrm{P}^{\infty}\right)$ as a module over the Steenrod algebra, Abstracts Amer. Math. Soc. 833 (1987), 55-89.

[11] D. V. Phuc and N. Sum, On the generators of the polynomial algebra as a module over the Steenrod algebra, C. R. Math. Acad. Sci. Paris 353 (2015), no. 11, 1035-1040.

[12] D. V. Phuc and N. Sum, On a minimal set of generators for the polynomial algebra of five variables as a module over the Steenrod alge- bra, Acta Math. Vietnam. 42 (2017), no. 1, 149-162.

[13] D. V. Phuc, $\mathcal{A}$-generators for the polynomial algebra of five variables and the Singer transfer, arxiv.org/abs/1810.06061v10.

[14] S. Priddy, On characterizing summands in the classifying space of a group, I, Amer. Jour. Math. 112 (1990), 737-748.

[15] J. Repka and P. Selick, On the subalgebra of $H_{*}\left((\mathbf{R P})^{n} ; \mathbf{F}_{2}\right)$ annihilated by Steenrod operations, J. Pure Appl. Algebra 127 (1998), no. 3, 273-288.

[16 ] W. M. Singer, The transfer in homological algebra, Math. Z. 202 (1989), no. 4, 493-523.

[ 17 ] J. H. Silverman, Hit polynomials and the canonical antiautomorphism of the Steenrod algebra, Proc. Amer. Math. Soc. 123 (1995), no. 2, 627637.

[18 ] N. E. Steenrod, Cohomology operations, Lectures by N. E. Steenrod written and revised by D. B. A. Epstein. Annals of Mathematics Studies, No. 50, Princeton University Press, Princeton, NJ, 1962.

[19] N. Sum, The negative answer to Kameko's conjecture on the hit problem, Adv. Math. 225 (2010), no. 5, 2365-2390.

[20 ] N. Sum, On the hit problem for the polynomial algebra, C. R. Math. Acad. Sci. Paris 351 (2013), no. 13-14, 565-568.

[ 21 ] N. Sum, On the Peterson hit problem, Adv. Math. 274 (2015), 432-489.

[ 22 ] N. K. Tín and N. Sum, Kameko's homomorphism and the algebraic transfer, C. R. Math. Acad. Sci. Paris 354 (2016), no. 9, 940-943.

[23 ] N. K. Tin, The admissible monomial basis for the polynomial algebra as a module over Steenrod algebra in some degrees, JP J. Algebra, Number Theory Appl. 46 (2020), no. 1, 55-68.

[24] N. K. Tin, The hit problem for the polynomial algebra as a module over Steenrod algebra, I, JP J. Algebra, Number Theory Appl. 47 (2020), no. 1, 67-86.

[ 25 ] G. Walker and R. M. W. Wood, Flag modules and the hit problem for the Steenrod algebra, Math. Proc. Cambridge Philos. Soc. 147 (2009), no. 1, 143-171.

[26 ] G. Walker and R. M. W. Wood, Polynomials and the mod 2 Steenrod algebra. Vol. 1, London Mathematical Society Lecture Note Series, 441, Cambridge University Press, Cambridge, 2018.

[27 ] G. Walker and R. M. W. Wood, Polynomials and the mod 2 Steenrod algebra. Vol. 2, London Mathematical Society Lecture Note Series, 442, Cambridge University Press, Cambridge, 2018.

[ 28 ] R. M. W. Wood, Steenrod squares of polynomials and the Peterson conjecture, Math. Proc. Cambridge Philos. Soc. 105 (1989), no. 2, 307-309. 